http://jmscr.igmpublication.org/home/

ISSN (e)-2347-176x ISSN (p) 2455-0450

crossref DOI: https://dx.doi.org/10.18535/jmscr/v8i12.39

Journal Of Medical Science And Clinical Research

\title{
A case report of stroke due to brain stem tuberculoma
}

\section{Dr Manomenane.M ${ }^{1}$, Dr A.K. Badrinath MD General Medicine², Dr S. Suresh Babu MD General Medicine ${ }^{3}$}

${ }^{1}$ Post Graduate, Department of General Medicine, Sri Manakula Vinayagar Medical College and Hospital

${ }^{2}$ Professor and Head of the Department, General Medicine, SMVMCH

${ }^{3}$ Assistant Professor, Department of General Medicine, SMVMCH

\begin{abstract}
Tuberculosis still highly prevalent in developing countries commonly affects the lungs. Central nervous system (CNS) involvement in tuberculosis is common in India but tuberculoma a manifestation of tuberculosis which mimics a tumor is not common. ${ }^{l}$ most of the CNS tuberculoma presents with seizures or focal signs. here we presents an elderly female with acute CVA with right sided hemiparesis .computed tomography of the brain showed a hyperdense lesion in the left pons. Further contrast enhanced MRI brain revealed left pontine tuberculoma. There are few case reports of tuberculoma presenting as acute stroke.
\end{abstract}

Keywords: Stroke, brain stem, tuberculoma.

\section{Introduction}

Tuberculosis a disease caused by acid fast mycobacterium tuberculosis complex bacteria commonly affect the lungs although other organs are involved in about one third of cases. In India with a huge burden of tuberculosis (incidence of 2.2 million cases in 2015) extra pulmonary tuberculosis is a common manifestation. ${ }^{1}$ CNS tuberculosis presents as basal meningitis which may complicate by causing obstructive hydrocephalus. Tuberculosis is a rare manifestation of CNS tuberculosis presenting as a space occupying lesion with focal signs or seizures. ${ }^{3}$

\section{Case Report}

A 67 years old female patient presented with sudden onset of difficulty in using right upper and lower limb for one day duration .she also had slurring of speech and urinary incontinence. No history of trauma, not a diabetic, hypertensive or coronary artery disease. No history of pulmonary tuberculosis in past or contact history of tuberculosis. On examination patient was conscious and oriented, pallor + , pulse -94 bpm and blood pressure was elevated 160/110mmhg. Cardiac and respiratory auscultation was normal and CNS examination revealed patient conscious and oriented, higher mental functions were intact. Patient had right UMN facial nerve palsy and urinary incontinence. Her power was $2 / 5$ in right upper and lower limb with hypertonia and exaggerated deep tendon reflexes, bilateral plantar extensor. Patient was admitted as a case of acute CVA with right hemiplegia with UMN facial palsy and evaluated. 
Her complete hemogram revealed anemia with HB- $8.4 \mathrm{gm} \%$. Her renal and liver function test was normal. ECG, chest $x$ ray and $2 \mathrm{D}$ echocardiography were normal .CT brain showed a ill defined hyperdense foci in the left side of pons with multiple calcific foci seen within the lesion suggestive of a space occupying lesion and planned for further evaluation. Patient was started on anti edema measures, anti hypertensives, physiotherapy and supportive care. contrast enhanced MRI brain revealed altered signal intensity lesion of size 1.4 x $1 \times 1.2 \mathrm{~cm}$ involving the left pons. The lesion shows hyperintense peripheral rim with hypointense centre. Post contrast study showed thick peripheral rim enhancement. Features were suggestive of granulomatous lesion probably TUBERCULAR GRANULOMA. Patient was started on category 1 anti- tuberculosis treatment and reviewed .patient had improvement in muscle power and repeat CT- brain after 6 months of treatment was normal

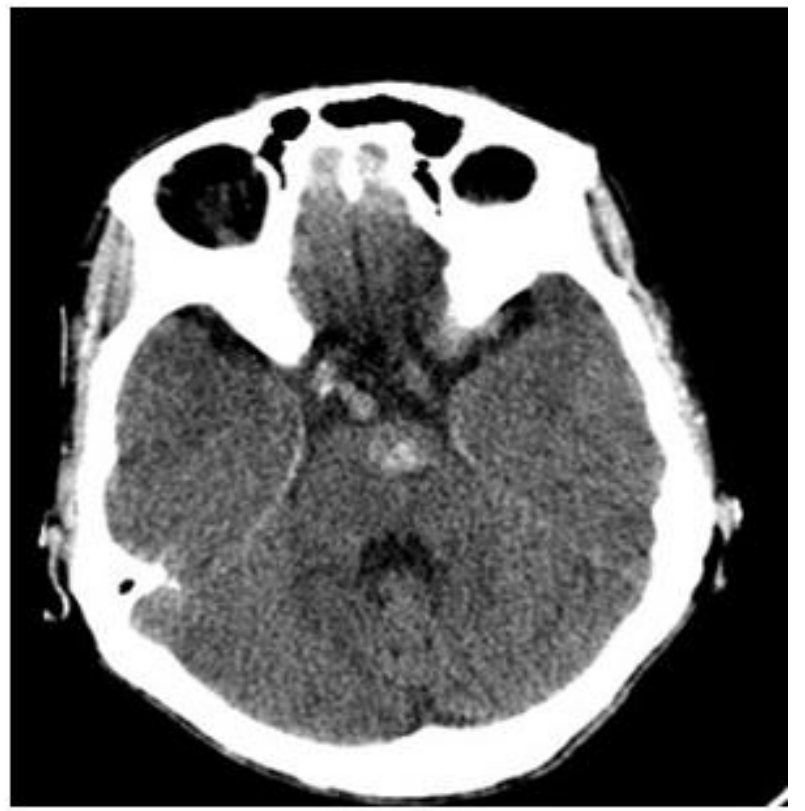

Figure 1: CT Brain plain of the patient showing a hyperdensefoci in the left side of pons with multiple calcific foci seen within the lesion

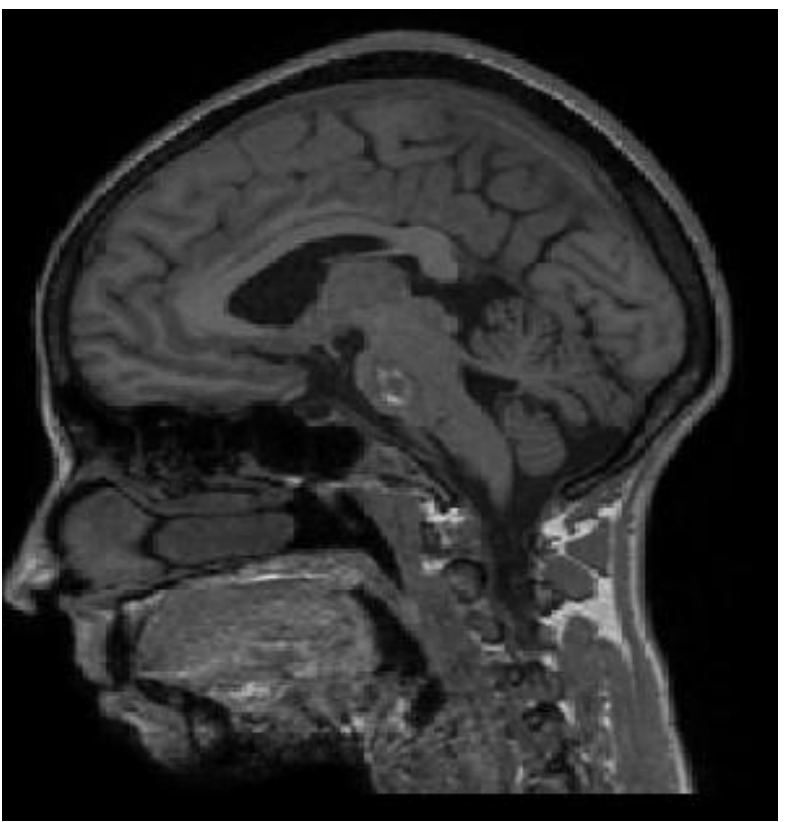

Figure 2: Contrast enhanced MRI brain revealed altered signal intensity lesion of size 1.4 x 1 x 1.2 $\mathrm{cm}$ involving the left pons.

\section{Discussion}

Tuberculosis usually affects the lungs but in India extra pulmonary manifestation of tuberculosis is high. CNS tuberculosis commonly manifests as meningitis and the incidence is high in developing countries with 20.6 per 1 lakh population. ${ }^{1}$ Most of the CNS tuberculosis were seen in extremes of age and with HIV infection. Still with highly effective anti tuberculosis treatment the morbidity and mortality remains high. ${ }^{2}$ Most of the cases results from hematogenous spread from a primary or post primary pulmonary tuberculosis. Tuberculoma is a manifestation of tuberculosis in which tubercles conglomerate to form a mass which mimics a tumor. CNS tuberculoma is an uncommon manifestation of tuberculosis and presents as a space occupying lesion. ${ }^{3}$ The exact pathogenesis behind formation of these tuberculoma is not understood. most of the CNS tuberculoma manifests as ring enhancing lesion involving the cerebral hemispheres which may be solitary or multiple. These CNS tuberculoma clinically manifests with seizures or focal signs. Brain stem tuberculoma is rare and there are few case reports in literature manifesting as one and half syndrome, gaze palsy or millard -Gubler 
syndrome. ${ }^{3}$ There are few case reports of brainstem tuberculoma (one case involving the pons and the other case involving the medulla) presenting as acute stroke as in our patient. Patient should be treated similar to other cases of tuberculosis with category -1 ATT drugs. Our patient improved symptomatically with ATT

To conclude CNS tuberculosis may present with atypical presentation as in our case as acute stroke (mimicking a hemorrhagic stroke). In high prevalent countries like India a high degree of suspicion is required to further evaluate such patients for early diagnosis and treatment to reduce morbidity and mortality. Contrast enhanced MRI and MR spectroscopy helps in definitive diagnosis of such patients.

\section{References}

1. Murthy JMK. Tuberculous meningitis: The challenges. Neurology India. 2010; 58(5): 716-22.

2. Rock RB, Olin M, Baker CA, Molitor TW, Peterson PK. Central nervous system tuberculosis:pathogenesis and clinical aspects. Clin Microbiol Rev.2008; 21(2):243-61.

3. Gautam VKS, Singh R, Khurana S. Brainstem tuberculoma presenting as stroke IOSR journals.2013; 4(6):18-19.

4. Ramamurthi B, Varadajan MG. Diagnosis of tuberculoma of the brain. Journal of neurosurgery. 1961;18;1-7.

5. Wasay M, Kheleani BA, Moolani MK, et al. Brain CT and MRI findings in 100 consecutive patients with intracranial tuberculoma. Neuroimaging. 2003;13 (3):240-47. 